\title{
Nonlinear bio-photonic crystal effects revealed with multimodal nonlinear microscopy
}

\author{
S.-W. CHU*, I.-H. CHEN*, T.-M. LIU*, C.-K. SUN*, S.-P. LEE $\dagger$,

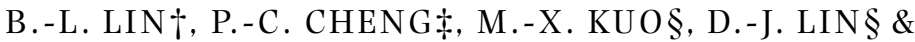 \\ H. - L. LIU § \\ *Department of Electrical Engineering \& Graduate Institute of Electro-Optical Engineering, National \\ Taiwan University, Taipei 10617, Taiwan, Republic of China \\ $\dagger$ Molecular \& Cell Biology Division, Development Center for Biotechnology, Taipei 10659, Taiwan, \\ Republic of China \\ $\ddagger$ Department of Electrical Engineering, State University of New York, Buffalo, NY 14260-2050, \\ U.S.A. \\ §Department of Physics, National Taiwan Normal University, Taipei 116, Taiwan, Republic of China
}

Key words. Cr: forsterite laser, multiphoton process, nonlinear microscopy, photonic crystal, second-harmonic generation, third harmonic generation.

\section{Summary}

Highly optically active nonlinear bio-photonic crystalline and semicrystalline structures in living cells were studied by a novel multimodal nonlinear microscopy. Numerous biological structures, including stacked membranes and aligned protein structures are highly organized on a nanoscale and have been found to exhibit strong optical activities through secondharmonic generation (SHG) interactions, behaving similarly to man-made nonlinear photonic crystals. The microscopic technology used in this study is based on a combination of different imaging modes including SHG, third-harmonic generation, and multiphoton-induced fluorescence. With no energy release during harmonic generation processes, the nonlinear-photonic-crystal-like SHG activity is useful for investigating the dynamics of structure-function relationships at subcellular levels and is ideal for studying living cells, as minimal or no preparation is required.

\section{Introduction}

Light microscopy, in particular fluorescence microscopy, has become extensively used in the study of the correlation between structure and function in modern biological sciences. Either intrinsic or extrinsic fluorescent probes are required in fluorescence microscopy using single- (Sheppard \& Shotton, 1997) or two-photon (Denk et al., 1990) excitation schemes.

Correspondence: C.-K. Sun. Tel.: +886223635251 ext. 319; fax: +8862 23677467; e-mail: sun@cc.ee.ntu.edu.tw
Hence, dye penetration, probe toxicity, and photo-bleaching/ damage are the most frequently encountered limiting factors. Nonlinear imaging modality, based on the nanostructure of the specimens, may provide an alternative tool in studying the dynamics of cellular structures and functions. Recent studies on man-made nano-periodic structures, e.g. super-lattices, indicate a strong enhancement in second-harmonic generation (SHG) (Zhao et al., 1999; Broderick et al., 2000; Dumeige et al., 2001) occurring only in noncentro-symmetric media. These demonstrated super-lattice structures are basically one-dimensional or two-dimensional nonlinear $\chi^{(2)}$ photonic crystals, defined as materials with a periodicity in second-order nonlinear dielectric properties (Berger, 1998). Similar to the group velocity effects in a $\chi^{(1)}$ photonic bandgap crystal that enhances the field intensity near the edge of the photonic bandgap, SHG conversion efficiency will be the highest when the $\chi^{(2)}$ spatial frequency is near the optical frequency and will not vanish even when the optical frequency is far away from the $\chi^{(2)}$ spatial frequency. Quasi-phase-matching is an example, as the operating wavelength is much smaller than the spatial modulation period of the nonlinear coefficient $\chi^{(2)}$. However, the nano nonlinear bio-photonic crystal in which we are most interested is another case when the operating wavelength is much longer than the spatial modulation period of $\chi^{(2)}$. A recent study on the enhancement of SHG by the existence of natural nano-photonic crystals, the bacteriorhodopsin (bR), using the Hyper-Rayleigh scattering method (Clays et al., 2001) has demonstrated the existence of a nonlinear photonic crystal effect (Berger, 1998) when the spatial modulation period $(5 \mathrm{~nm})$ is much smaller than a fraction of the investigating optical wavelength (1064 nm). Therefore, it 
is reasonable to speculate that most of the highly organized biological nano-structures may all break optical centrosymmetry and behave as SHG-active nonlinear photonic crystals. Examples of highly organized biological nano-structures include stacked membranes, the myelin sheath, endoplastic reticulum (ER), grana in the chloroplast, Golgi apparatus, cellulose microfibrils and collagen bundles. It is highly possible that these natural biological structures behave as nonlinear bio-photonic crystals with enhanced SHG activities. The use of SHG to image the nano nonlinear bio-photonic crystals should thus provide a new imaging modality for threedimensional (3D) imaging of living cells due to the fact that most bio-molecules are highly asymmetric and that randomly arranged bio-molecules behave like an amorphous material with a diminished SHG effect. In this paper we describe a study using multimodal nonlinear microscopy (Chu et al., 2001) to investigate nonlinear optical activities in naturally occurring bio-photonic crystalline structures. This novel microscopic technique, in conjunction with the use of a $\mathrm{Cr}$ : forsterite laser, is based on a combination of different imaging modalities, including second-, third-harmonic generations, and multiphoton fluorescence.

Similar to two-photon fluorescence (2PF) microscopy (Denk et al., 1990), SHG microscopy (Gannaway \& Sheppard, 1978) provides superior axial resolution due to the quadratic dependence of the signals on the illumination intensity. Because SHG does not occur in optically isotropic media, SHG microscopy was first demonstrated for the studies of SHG photonic crystals (Gannaway \& Sheppard, 1978), surfaces/interfaces (Shen, 1989), field distribution in semiconductors (Sun et al., 2000, 2001), and was then applied to biological study, including membrane potentials (Campagnola et al., 1999; Peleg et al., 1999; Moreaux et al., 2000) and tissue polarity (Freund et al., 1986; Guo et al., 1997). Because of optical isotropy created by randomly organized biological structures, no SHG is expected if there is no organized structure or other cause to break centro-symmetry in the optical scale. Here we found that SHG microscopic images correspond to specially organized crystalline nano-structures in biological samples where optical centro-symmetry is broken. In contrast to laser-induced fluorescence processes, only virtual states are involved in harmonic generations. The marked advantage of this virtual transition during wavelength conversion is the lack of energy deposition, thus no photo-damage or bleaching from the process is expected, and the technique can be regarded as a truly 'non-invasive' imaging modality.

Because SHG arises from the nano-crystalline structures under study, it is highly desirable to use other non-invasive harmonic generation effects to image general cellular features so that proper correlation of structures can be obtained. Because of the optical dispersion property in biological tissues, third-harmonic generation (THG) was proven to be generated from regions with optical inhomogeneity and was applied to image general cellular structures (Barad et al., 1997; Müller et al., 1998). With third power dependence on the illumination intensity, THG provides even better optical sectioning resolution than SHG or 2PF using the same illumination wavelength, but is more sensitive to attenuation of the illumination light. Here we use THG microscopy for general interface imaging, in comparison to SHG microscopy of nano-structures.

\section{Experimental details}

\section{Light source selection}

In order to allow both SHG/THG to fall within the visible spectrum and also provide advantages of low illumination attenuation, we moved the excitation wavelength to a 1200$1350 \mathrm{~nm}$ regime by using a $\mathrm{Cr}$ : forsterite laser. The most common light source for multiphoton excitation microscopy now is the femtosecond Ti: sapphire laser with an emission wavelength around $800 \mathrm{~nm}$. For the purpose of SHG microscopy or $2 \mathrm{PF}$ microscopy, the wavelength of signals excited by a Ti: sapphire femtosecond laser will be around the blue edge (SHG) of, or inside (2PF), the visible wavelength region. For THG microscopy, however, the wavelength of THG signals will fall into ultraviolet (UV) region. Consequently, these THG signals will suffer strong UV absorption of most biological specimens (Cheng et al., 2001; Lin et al., 2001) and will decay quickly along the optical path inside the specimens. At the same time, special UV optical components are required, such as objectives and mirrors, which are often much more expensive than their counterparts designed for visible light. In order to make THG microscopy routinely achievable, we need to move the pumping wavelength longer than $1200 \mathrm{~nm}$. By using a Cr: forsterite laser with centre wavelength around $1230 \mathrm{~nm}$, not only the visible but also the near-infrared (NIR) spectrum is now open for simultaneous recording of $2 \mathrm{PF}$, three-photon fluorescence (3PF), SHG and THG. Our previous studies using $1230 \mathrm{~nm}$ light indicated efficient excitation of $2 \mathrm{PF}$ and $3 \mathrm{PF}$ in common bio-probes (Liu et al., 2001).

Figure 1 shows that light attenuation (including absorption and scattering) spectra of the epidermal layer on an onion bulb scale (Fig. 1A), porcine skin (Fig. 1B) and the grey matter of porcine brain (Fig. 1C). In the near-UV wavelength region, the attenuation is dominated by the strong absorption of common tissue constituents such as proteins and carbohydrates. For an IR wavelength longer than $1400 \mathrm{~nm}$, however, the molecular resonant absorption of water starts to dominate the attenuation. As a result, a biological specimen has a transparent window between 400 and $1300 \mathrm{~nm}$. This is one of the reasons why a Ti: sapphire laser, whose centre wavelength is around $800 \mathrm{~nm}$, is widely adopted as the light source in multiphoton microscopy. By observing the attenuation spectra (absorption and scattering), however, one finds that the attenuation minimum falls around $1200-1300 \mathrm{~nm}$, instead of $800 \mathrm{~nm}$. In fact, the observation is not surprising because the scattering cross-section decreases for longer wavelengths. 

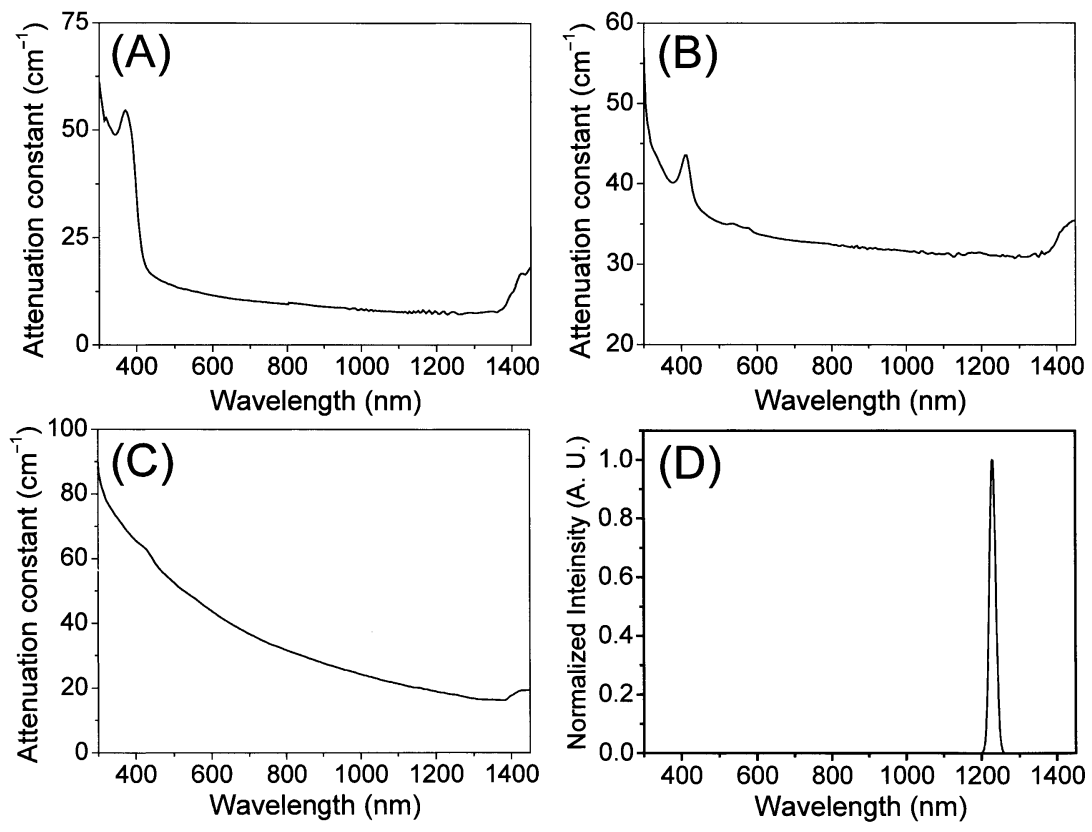

Fig. 1. Light attenuation (absorption and scattering) spectra in the (A) epidermal cells on onion bulb scale, (B) porcine skin, and (C) the grey matter of porcine brain. (D) Spectrum of Cr: forsterite laser. Note the wavelength lies within the transparency window of most biological specimens.
This is also in good agreement with previous measurements of the attenuation coefficients of human skin (Anderson \& Parrish, 1981), maize stem (Cheng et al., 1998) and leaf (Lin et al., 2001). The developed nonlinear microscope based on a Cr: forsterite laser thus allows us to utilize this transparent window in most biological specimens. The spectrum of the $\mathrm{Cr}$ : forsterite laser is shown in Fig. 1(D) with a centre wavelength around $1230 \mathrm{~nm}$. Because of the low attenuation coefficient at this wavelength, only one order of magnitude reduction in the signal (for SHG and $2 \mathrm{PF}$ ) was observed at a depth of $360 \mu \mathrm{m}$ into a maize stem fixed in 10\% ethanol (Chu et al., 2001), in good agreement with previous light attenuation measurement of maize stems (Cheng et al., 1998). Comparing the attenuation spectra of plant tissues (onion or maize stem) with that of a porcine brain (Fig. 1C), we can expect significantly better depth penetration performance of $\mathrm{Cr}$ : forsterite laser source over the Ti: sapphire laser in brain slice research. This superior depth performance agrees well with previous studies for optical coherent tomography (Bouma et al., 1996) comparing penetration depth between 800 and $1300 \mathrm{~nm}$ light sources. Moreover, due to the high absorption in the visible and near-infrared (NIR) spectrum in plant materials, this wavelength is particularly useful for imaging plant specimens. Significant reduction in photo-induced damage in plant materials was observed by conducting $2 \mathrm{PF}$ microscopy using a femtosecond $\mathrm{Cr}$ : forsterite laser compared with a femtosecond Ti: sapphire laser under similar illumination intensity and exposure (Chen et al., in press).

\section{Experimental set-up}

Figure 2 shows the experimental set-up of the multimodal nonlinear microscope. A home-built $\mathrm{Cr}$ : forsterite laser, which operates at $1230 \mathrm{~nm}$ with a repetition rate of $110 \mathrm{MHz}$ and a pulse width of $100 \mathrm{fs}$ ( $350 \mathrm{~mW}$ average output), was used as the light source (Liu et al., 2001). The laser output was first shaped and collimated by a telescope to fit the aperture of the focusing objective. The collimated beam was then coupled into an Olympus BX30 microscope through a reflected light fluorescence attachment (BX-FLA) with a dichroic beamsplitter (Chroma 970dcspxr) to reflect the IR laser and transmit visible light with $45^{\circ}$ incident angle. The excitation laser light was focused onto the biological sample with a spot size close to diffraction limit by high NA objectives (ULWD MIR 80×/NA 0.75 or UPlanApo $60 \times /$ NA 1.20, Olympus) and the excited photoemission spectra were collected using an opposing objective. The fundamental IR beam was removed with an infrared blocking filter. The collected light (visible and NIR) was then directed into a spectrometer (SpectraPro-150, Roper Scientific, Trenton, NJ, USA) and recorded by a TE-cooled CCD detector (DV42-0E, Andor Technology, Belfast, UK). A mechanical scanning stage was used to achieve raster scan in order to obtain $x-y-z-\lambda$ images with detailed nonlinear spectrum corresponding to each pixel recorded (the $x-y$ plane is defined as the plane perpendicular to laser propagation direction). On the other hand, the laser spectrum was taken with a spectrometer (74000, Oriel Cornerstone, Stratford, CT, USA) equipped with an InGaAs photodiode (DET410, Thorlabs, Newton, NJ, USA). Attenuation spectrum was measured with a spectrophotometer (Lambda 900, Perkin Elmer, Wellesley, MA, USA).

\section{Results and discussion}

The cell wall of higher plants consists of macrofibrils, which are bundles of orderly arranged cellulose microfibrils with a diameter about $10 \mathrm{~nm}$. Within the microfibrils, there are micelles, representing another degree of highly ordered crystalline 
Fig. 2. Experimental set-up of a multimodal nonlinear laser scanning microscope. Laser: femtosecond $\mathrm{Cr}$ : forsterite laser centred at $1230 \mathrm{~nm}$; BS: beamsplitter; CA: camera; L: mercury lamp; S: sample; TS: threedimensional translation stage; F: colour filter; SM: spectrometer; CCD: charge-coupled device; C: computer.

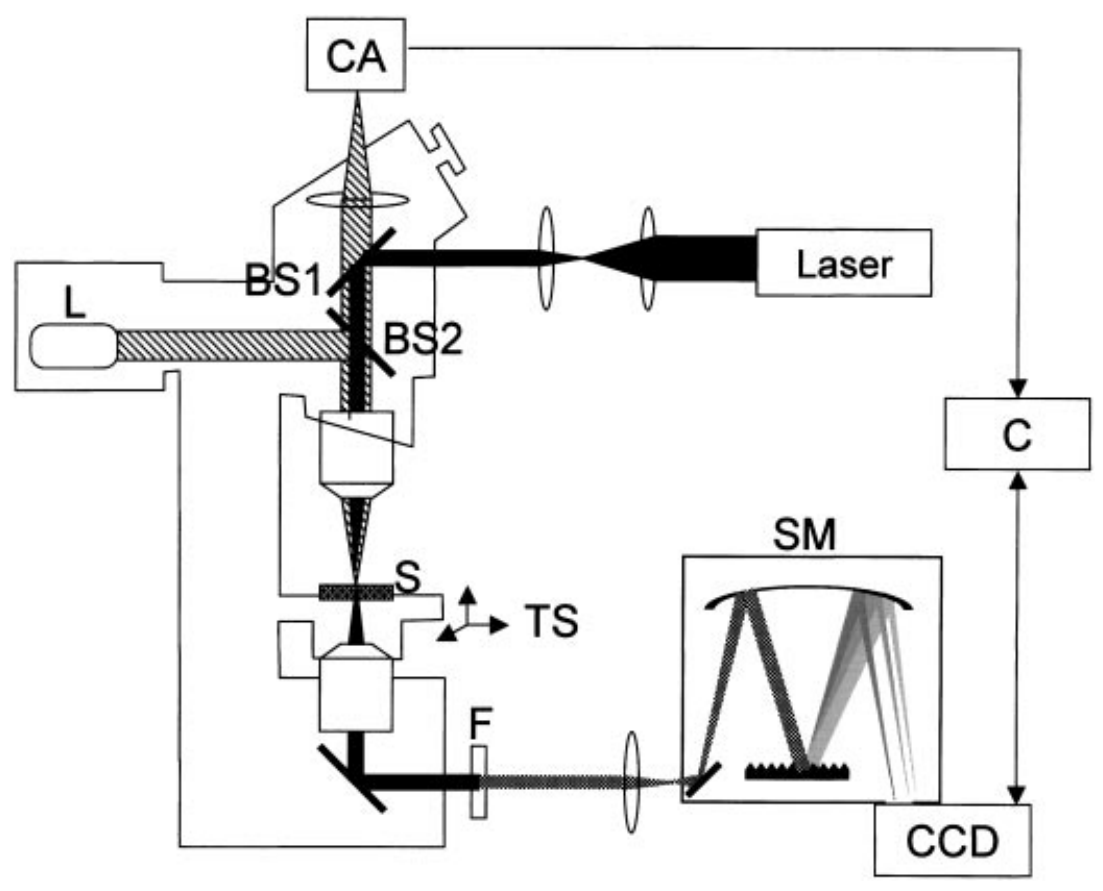

structure (Peter et al., 1992). These photonic-crystal-like structures produce optical anisotropy and provide active SHG. Figure 3(A) shows the nonlinear emission spectra measured from the cell wall of a parenchyma cell in maize (Zea mays L.) stem. Symmetric THG and SHG spectra centred at 410 and $615 \mathrm{~nm}$ can be observed, with intensity similar to, or stronger than, the residual $2 \mathrm{PF}$ centred at $680 \mathrm{~nm}$. Strong THG is induced due to optical inhomogeneity within and surrounding the cell wall, whereas SHG reflects the highly organized crystalline structures that break the 3D optical centro-symmetry in the wall. The source of SHG is further confirmed by the strong SHG signal obtained from the stone cell of pear (Pyrus serotina R.) (Fig. 3B). The extensive secondary wall development of the sclerenchyma cell generates significant SHG signals.

The starch granule, a highly birefringent structure, consists of crystalline amylopectin lamellae organized into effectively spherical blocklets and large concentric growth rings (Gallant et al., 1997). These optically active structural features are presumably responsible for the strong SHG that we observed in Fig. 3(C). For example, the SHG signal from a potato (Solanum tuberosum L.) starch granule is so strong that is visible to the naked eye, even under ambient room light (as shown in Fig. 4). The crystalline lamellae in starch granules are believed to consist of ordered double-helical amylopectin sidechains and are interleaved with more amorphous lamellae consisting of the amylopectin branching regions. The amylopectin side chain clusters within the crystalline lamellae have varying sizes but, on average, are around $10 \mathrm{~nm}$ wide by $9-$ $10 \mathrm{~nm}$ long (the length represents the thickness of the lamellae). These orderly arranged nano-layers form the bio-photonic structure, breaking the centro-symmetry and generating strong SHG (Mizutani et al., 2000). On the other hand, there are some larger structures in starch granules made up of crystalline hard shells and semicrystalline soft shells hundreds of nanometres in size. These alternating crystalline and semicrystalline rings with spatial modulated nonlinear properties could behave as 3D nonlinear photonic 'bandgap' crystals (Berger, 1998). The unexpectedly strong SHG activity might not only be the result of its super-helical amylopectin nanostructures, but could also suggest possible field enhancement with group velocity effect, similar to the density of state enhancement effect near the edge of the $\chi^{(1)}$ photonic bandgap. The visible SHG intensity might also suggest a possible SHG non-collinear phase-matching condition provided with its reciprocal lattice basis vectors of the 3D photonic bandgap crystals. The spatial frequency of high-order structures from the order of $200 \mathrm{~nm}$ up to the order of $10 \mu \mathrm{m}$, depending on illumination wavelength and composition materials, could all provide the non-collinear phase matching base-vector for the SHG process and can be considered as nonlinear bio-photonic bandgap crystals, providing even stronger SHG activity.

Compared with the emission spectra excited by a femtosecond Ti: sapphire laser in these plant specimens, the intrinsic 2PF signals are greatly reduced by using a femtosecond $\mathrm{Cr}$ : forsterite laser as the pumping source (Chu et al., 2001), while the probability of optical nonlinear damage is also greatly reduced (Chen et al., in press). As an example, Fig. 5 shows a quantitative comparison between the emission spectra excited by a $150 \mathrm{~mW} 1230 \mathrm{~nm} \mathrm{Cr}$ : forsterite femtosecond laser (120 fs pulsewidth, solid line), a $50 \mathrm{~mW} 780 \mathrm{~nm}$ Ti: sapphire femtosecond laser ( $120 \mathrm{fs}$ pulsewidth, dashed line), and a $0.45 \mathrm{~mW} 365 \mathrm{~nm}$ UV light source from a frequency-doubled Ti: sapphire laser (dotted line). The emission spectra from the cell wall of an epidermal cell in a rice leaf (Oryza sativa) and of the 

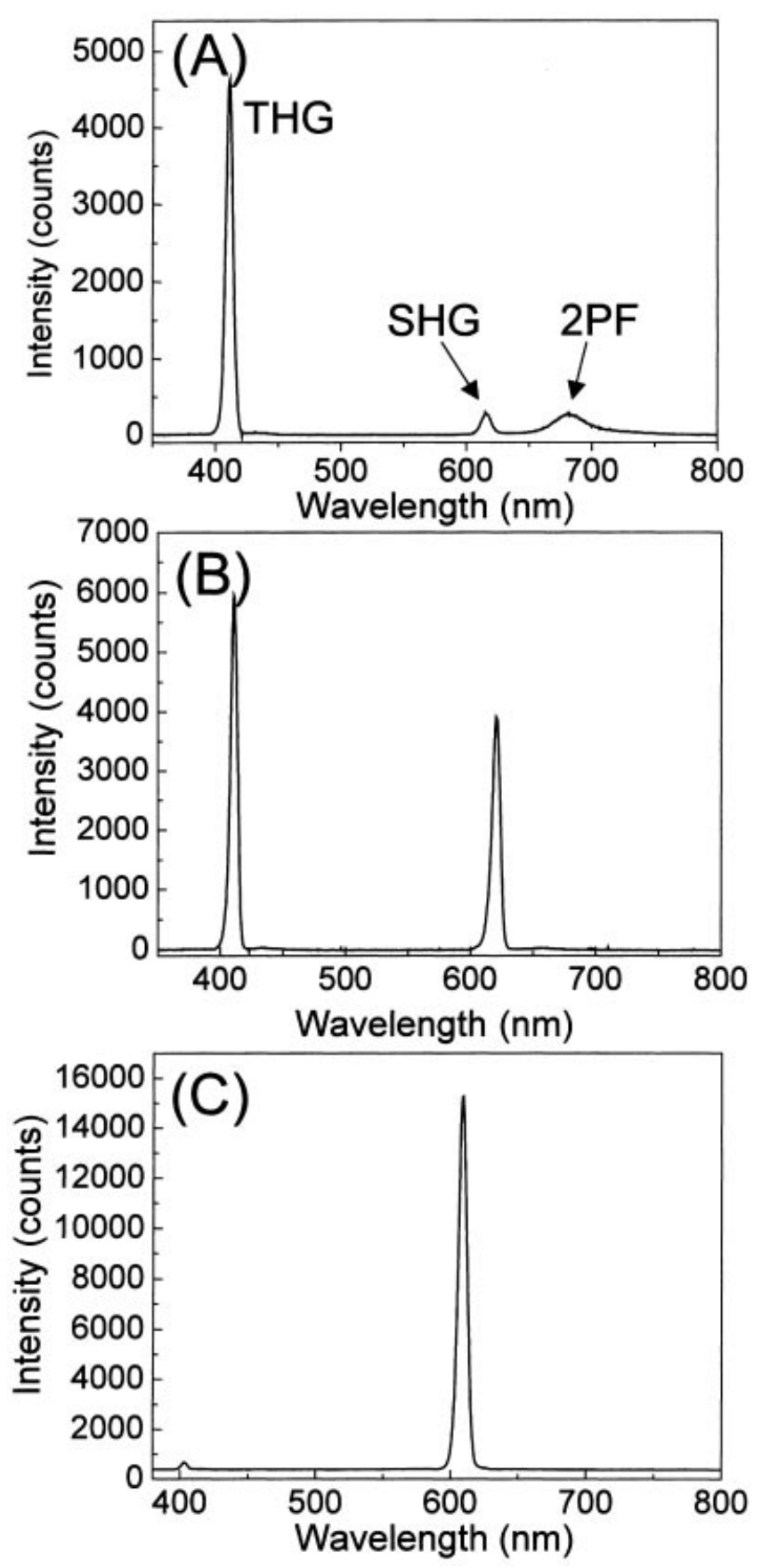

Fig. 3. Nonlinear emission spectra measured from (A) the cell wall of a parenchyma in maize (Zea mays) stem, (B) the stone cell of a pear (Pyrus serotina R.) fruit, and (C) potato (Solanum tuberosum L.) starch granule. These spectra were recorded under similar illumination intensity, with normalized 0.1-s integration time. Note the extraordinarily strong SHG from the starch granule.

parenchyma cells in a maize stem are shown in Fig. 5(A) and (B), respectively. Broad autofluorescence covering the whole visible and NIR region is evident with both UV (single-photon fluorescence) and Ti: sapphire excitation (2PF), whereas with $1230 \mathrm{~nm}$ excitation, only weak residue $2 \mathrm{PF}$ and efficient
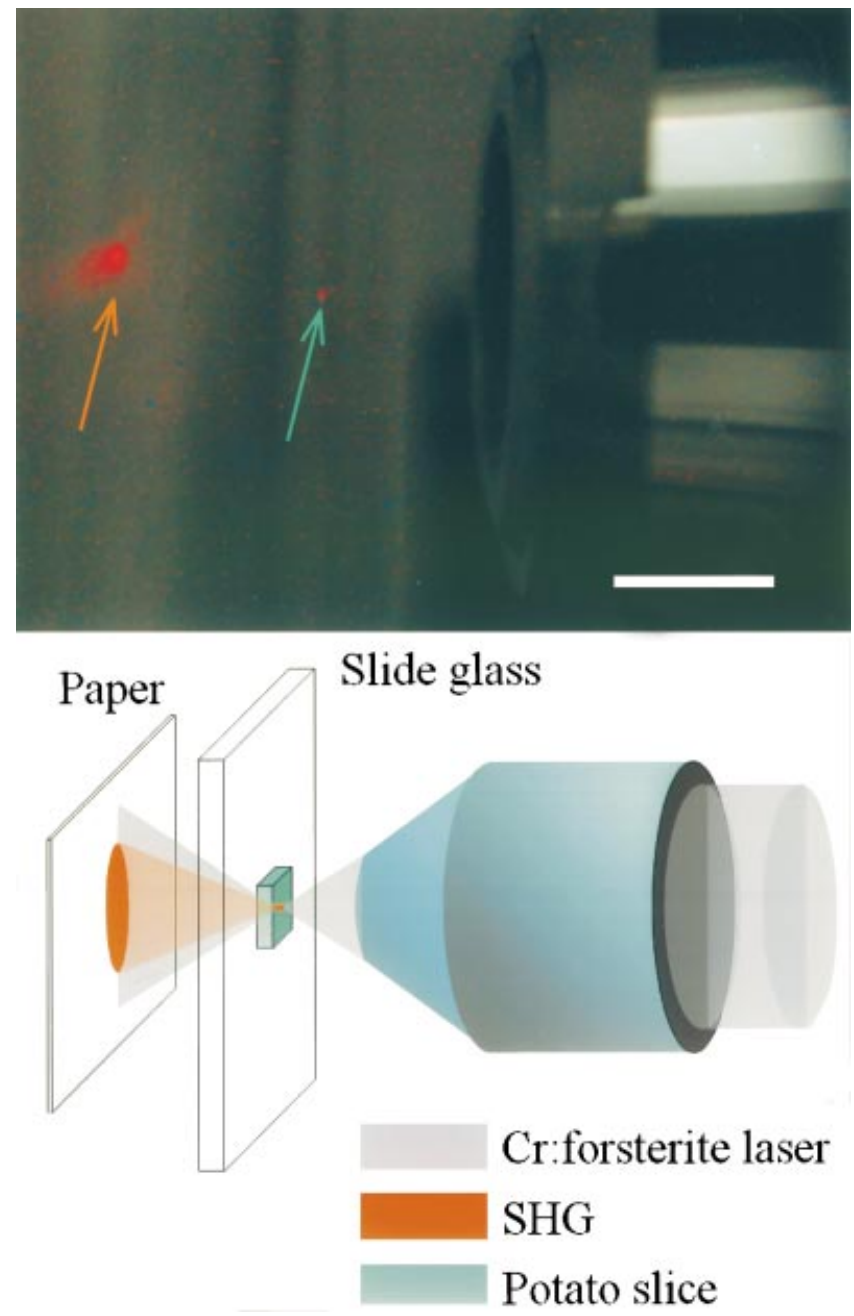

Fig. 4. Photograph showing the generated red SHG from a thin potato slice (the green arrow) on a white paper (the orange arrow) under a $\mathrm{Cr}$ : forsterite laser excitation (at a wavelength of $1230 \mathrm{~nm}$ ). This photograph was taken with a conventional camera, which is not sensitive to the excitation laser wavelength. The corresponding set-up is shown schematically at the bottom of the figure. Scale bar: $5 \mathrm{~mm}$.

harmonic generations are observed. With suppressed background autofluorescence using longer wavelength excitation, the whole visible and NIR region can then be left for efficient multiphoton fluorescence dye to label different functional molecules. At the same time, because of the suppressed fluorescence, the unfavourable energy deposition due to energy difference between the absorbed and emitted photons is also suppressed, resulting in a lower probability of induced photodamages. Furthermore, because there is no energy deposition at all during harmonic generation processes, no photo-damage effect is expected with SHG and THG. Thus, with efficient SHG and THG signals, along with the reduced but still measurable intrinsic $2 \mathrm{PF}$ signals, the femtosecond $\mathrm{Cr}$ : forsterite laser appears to be one of the best choices for intrinsic multimodal microscopy, 
Fig. 5. Nonlinear emission spectra from the cell wall of (A) an epidermal cell in rice leaf and (B) parenchyma cells in maize stem with three different sources: UV light (dotted line), Ti: sapphire laser (dashed line), and Cr: forsterite laser (solid line). The suppression of background 2PF and the symmetric peaks of THG and SHG in Cr: forsterite excitation spectrum are evident.
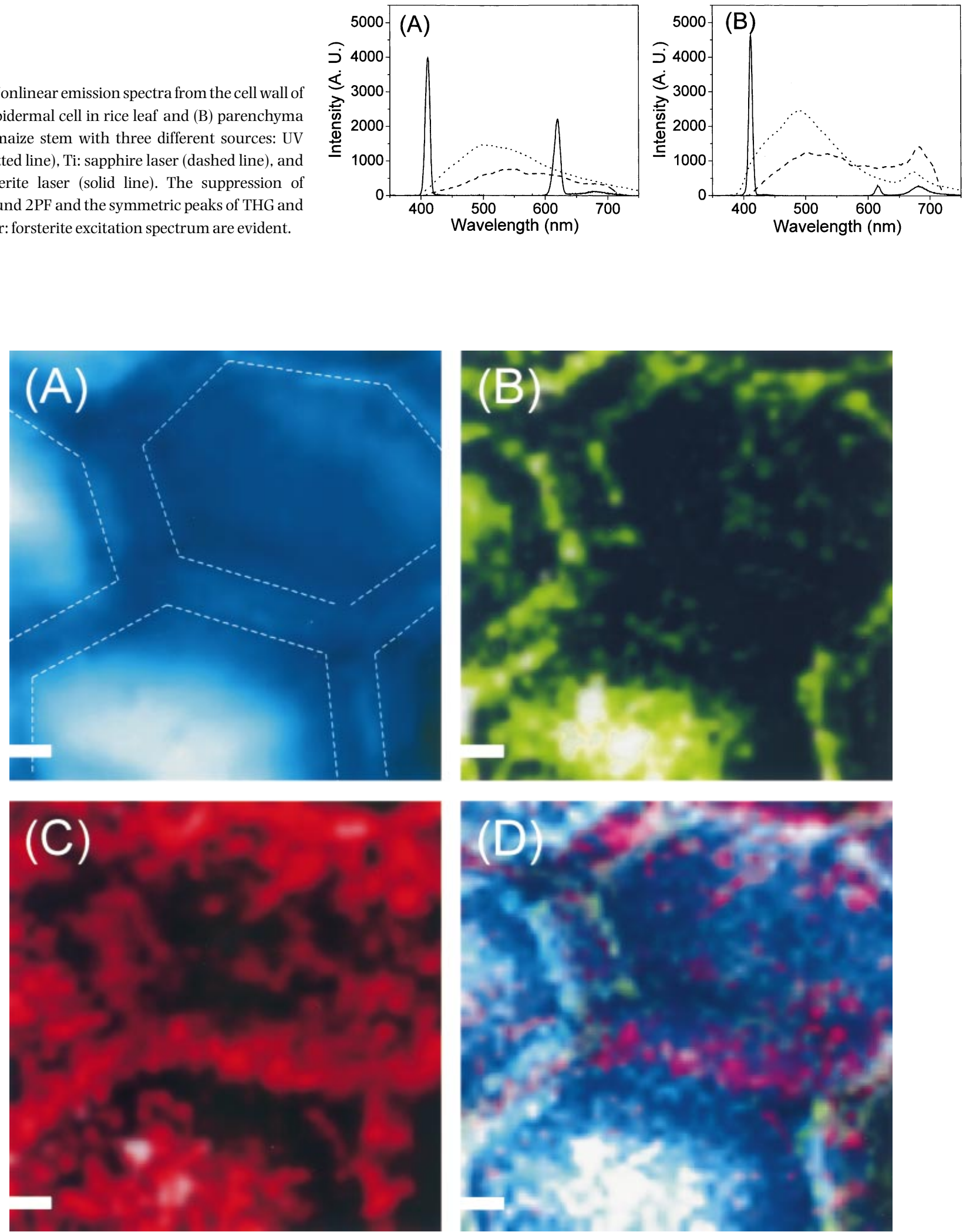

Fig. 6. Scanning $x-y$ - $\lambda$ images corresponding to the wavelength of (A) THG (shown in blue), (B) SHG (shown in green), and (C) 2 PF (shown in red) in the parenchyma cells of a maize stem (profiled by dashed line). (D) The combined multimodal image showing relative sources of different signals, which can provide useful information on the structure-function relationship. Scale bar: $20 \mu \mathrm{m}$. 

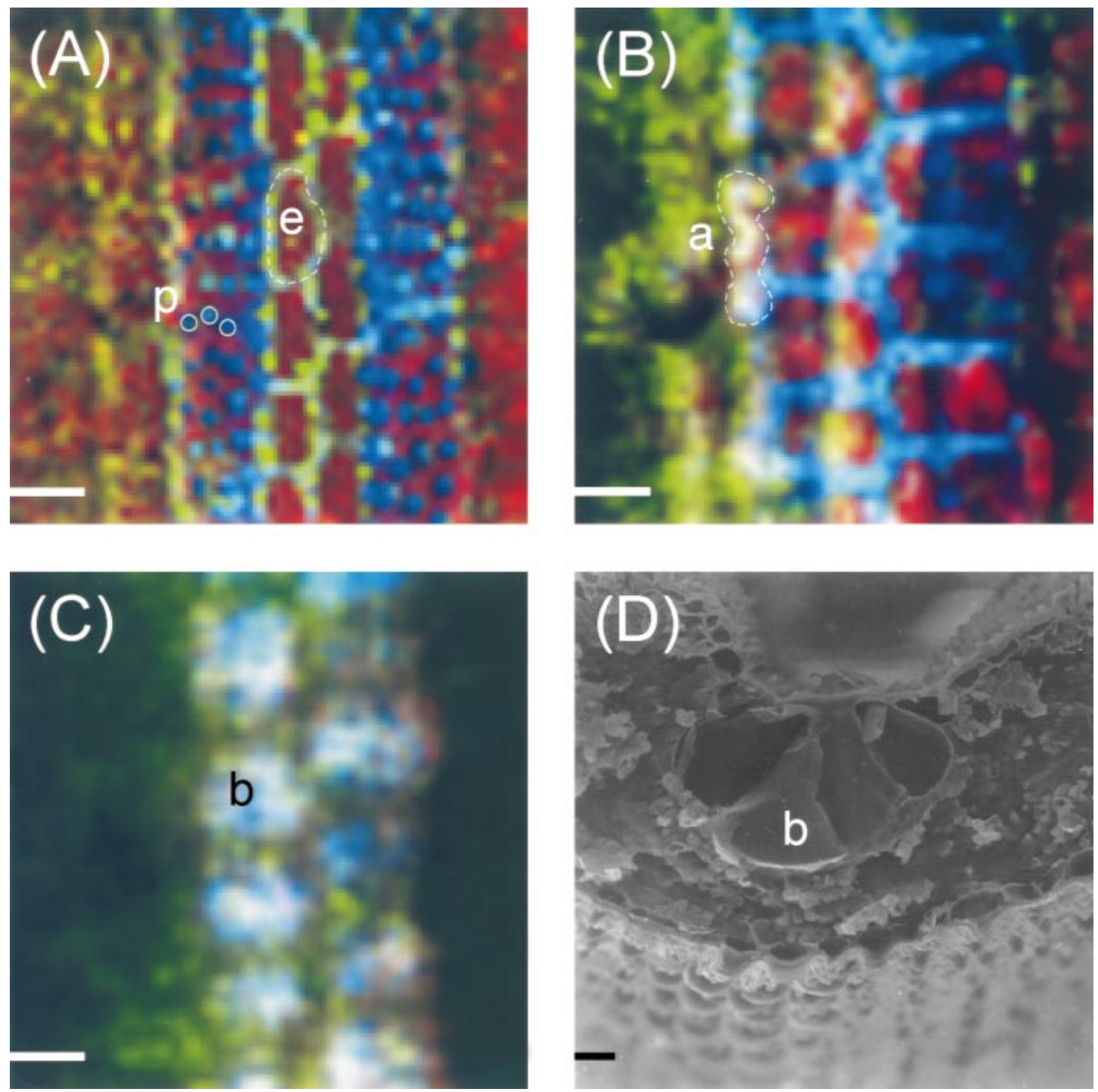

Fig. 7. Scanning multimodal $x-y$ - $\lambda$ images from the adaxial surface of a rice (Oryza sativa L.) leaf at different depths: (A) $10 \mu \mathrm{m}$, (B) $20 \mu \mathrm{m}$, and (C) $40 \mu \mathrm{m}$ from the sample surface (1, 2 and 3 represent THG, SHG and 2PF images, respectively). (D) SEM image of a cross-sectional view of a similar rice leaf. Scale bar: $15 \mu \mathrm{m}$. e: epidermal cell, p: papillae, a: armed-type parenchyma cell, b: bulliform cell.

combining these three different signals while the whole visible spectrum is left for extrinsic multiphoton dye labelling.

Figure 6 shows the specific $x-y$ - $\lambda$ images corresponding to THG, SHG and 2PF in the parenchyma cells of a maize stem taken at a depth of $110 \mu \mathrm{m}$ from the sample surface. The sample thickness was around $500 \mu \mathrm{m}$. With an average power of $100 \mathrm{~mW}$ before entering the sample and a focused spot size of $1.3 \mu \mathrm{m}$, the intensity at focus is in the range $9-50 \times 10^{10}$ $\mathrm{W} \mathrm{cm}{ }^{-2}$, depending on the focal depth inside the sample. As expected, strong SHG and THG can be observed in the cell wall. THG shows the longitudinal cell walls in the centre of the image and the transverse cell walls of several adjacent cells (Fig. 6A), revealing the ability of THG to pick up the whole cell profile. SHG shows the relative position of the orderly structured secondary walls through bio-photonic effects. In the region with extensive secondary wall growth, the separation of the secondary wall can be clearly observed in Fig. 6(B). By contrast, 2PF signals indicate the distribution of the functional molecules with auto-fluorescence (Fig. 6C). By comparing different modality images, the co-localization of different signals can be shown, which will be useful in studying the relationship between bio-structure (harmonic generations) and molecule functions (two- and multiphoton fluorescence) in various biological tissues. More than $500 \mu \mathrm{m}$ depth penetration in the maize stem sample was achieved with the $\mathrm{Cr}$ : forsterite laser (Chu et al., 2001).

A paradermal optical section of the adaxial surface of a rice (Oryza sativa L.) leaf is shown in Fig. 7. From these multimodal 
Fig. 8. (A) Strong SHG emission recorded from mouse skeletal muscle fibres. (B) Longitudinal THG and (C) SHG optical sections of a mouse skeletal muscle fibre, respectively. Individual sacromeres can be observed in the SHG scanning image. (D) The combined image. Scale bar: $15 \mu \mathrm{m}$. sa: sacromere.

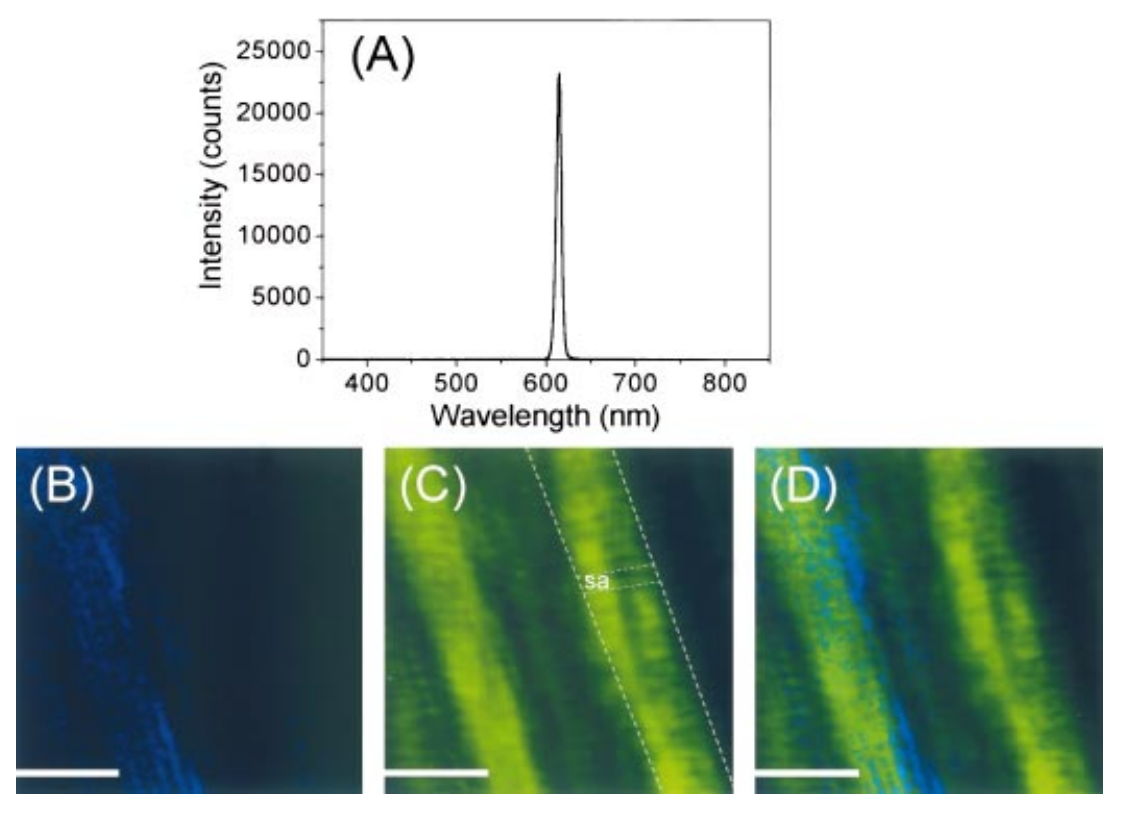

images, THG picks up structural interfaces, such as the papillae from the cuticular layer and the cell wall of bulliform cells. SHG reflects bio-photonic structures as expected, including the cuticular papillae and longitudinal cell walls, because of the orderly arranged cutin, waxes and cellulose microfibrils, respectively. $2 \mathrm{PF}$, by contrast, picks up the fluorescent chromophores. For correlation purposes, Fig. 7(D) also gives a SEM micrograph showing the cross-sectional view of a similar rice leaf.

There are a number of structures in animal tissues that are also good candidates for a strong bio-photonic crystalline effect. For instance, the sacromeres in skeletal muscles are composed of crystalline myosin and actin nano-filaments with 40 and $20 \mathrm{~nm}$ periods, respectively, falling into the spatial range of strong SHG activity. Figure 8 shows longitudinally sectioned $x-y-\lambda$ images obtained from mouse skeletal muscle. The SHG intensity fluctuation within one sarcomere, which provides the striped features in Fig. 8(C), reflects the structure difference between the A-band and I-band on the arrangement of actin and myosin nano-filaments. By contrast, the weak THG picks up the longitudinal interface of myofibril and surrounding cytoplasm due to induced optical inhomogeneity. The strong SHG activity from the actin/myosin complex can be a useful tool for the study of muscle cell dynamics.

The strength of the SHG signals that reflect the bio-photonic effects can vary according to the orientation of the organized nano-structures, which may have different $\chi^{(2)}$ matrix components. This mechanism provides the opportunity for structural orientation studies using SHG with controlled illumination polarization. For instance, the dumb-bell-shaped silica deposition in rice leaf also produces significant SHG signals. By varying the incident light polarization (shown as arrows in Fig. 9), the concentrically deposited silica layers in the silica cells of rice leaf (Hodson and Sangster, 1989) produce SHG images with a particular orientation in respect to that of the illumination polarization. In contrast, no polarization dependency was found in THG images. For comparison, a paradermal SEM image shows silica cells taken from a similar rice leaf (Fig. 9E).

Because of the lower probability of photo-damage with multimodal microscopy, one major advantage of such novel technique is the ability to inspect biological tissues or cells in vivo. Figure 10 shows the $x-y-\lambda$ scanning images of a live mesophyll cell from Commelina communis L. 2PF signals are generated from the highly auto-fluorescing photosynthetic pigments, such as chlorophyll (Fig. 10C). THG and SHG, however, seem to appear in different suborganelle compartments (Fig. 10A and B). To identify the source of these harmonic signals, a higher numerical aperture objective was used to provide better resolution. The enlarged scanning images of individual chloroplasts are shown in Fig. 10(E)-(H). The distribution of chlorophyll inside the chloroplasts is shown by 2PF signals, whereas THG provides information on the various sub-organellar interfaces. SHG signals, by contrast, correspond to the nano-organized bio-photonic structures in the chloroplast. By matching the SHG image with TEM images of similar specimens (data not shown), we concluded that the signals of SHG are the result of the orderly stacked thylakoid membranes in grana (crescent shaped) and the highly birefringent starch granules (oval or round shaped) in the chloroplasts (Gunning \& Steer, 1996). The stacked thylakoid membranes of grana provide the structural requirement for efficient SHG resembling the behaviour of photonic crystals.

\section{Summary}

Our results reveal nonlinear photonic crystal-like optical activity in living cells using multimodal microscopy. Highly organized nano-periodic structures in biological samples exhibit strong SHG activities resembling nonlinear photonic 

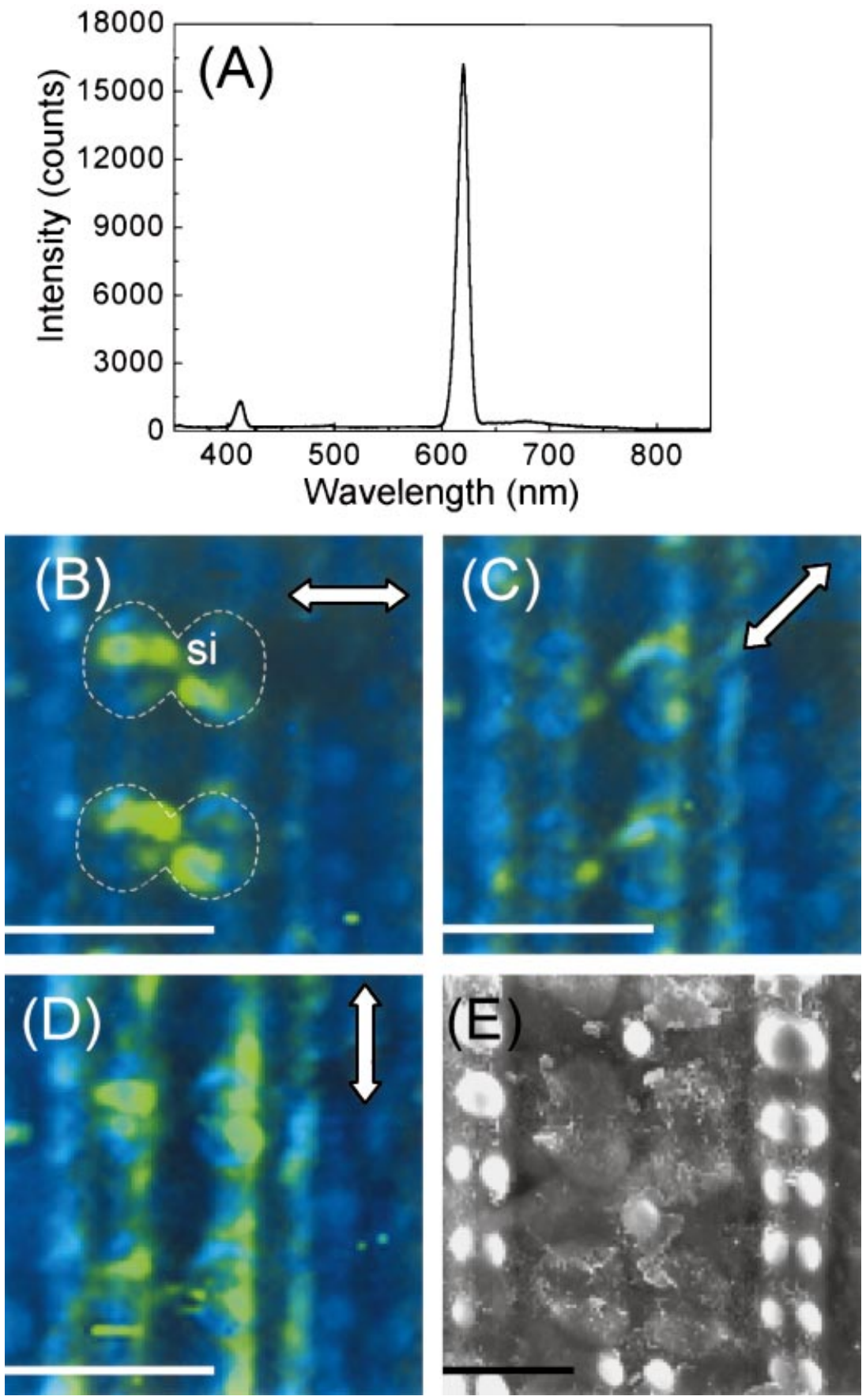

Fig. 9. (A) The emission spectrum from the silica deposition on the adaxial epidermis of rice leaf. (B)(D) Scanning $x-y-\lambda$ images of the adaxial surface of a rice leaf showing dumb-bell-shaped silica cells (profiled by dashed line). The illumination polarization is shown as arrows in each image. The orientation of SHG images follows the illumination polarization, whereas no correspondence can be found in THG images. A paradermal SEM image is given in (E) for comparison. Scale bar: $15 \mu \mathrm{m}$. si: silica cell. crystals and thus can be treated as 'nonlinear bio-photonic crystals'. A number of biological structures exhibit strong bio-photonic effects, such as microfibrils in cell walls, alternative crystalline lamellae in starch granules, cuticular papillaes on the epidermal cells of rice leaves, crystalline myosin and actin nano-filaments in the myofibrils of skeletal muscles, and grana in chloroplasts. The relative positions of these bio-photonic structures can be examined using SHG microscopy, whereas the interfaces and the functional molecules can be separately located by THG and 2PF microscopies. In contrast to single- and multiphoton absorption, harmonic generations involve only virtual states without energy deposition, thus minimal photo-damage occurs during these processes. The harmonic generations allow structural visualization with minimal or no additional preparation of the samples. Meanwhile, 2PF imaging modes should be included to monitor multiple molecular probes in live cells and tissues, such as those from transformed cells and transgenic organisms. Multimodal microscopy can provide a powerful tool for investigating the dynamics of structurefunction relationships at the molecular and subcellular levels. 

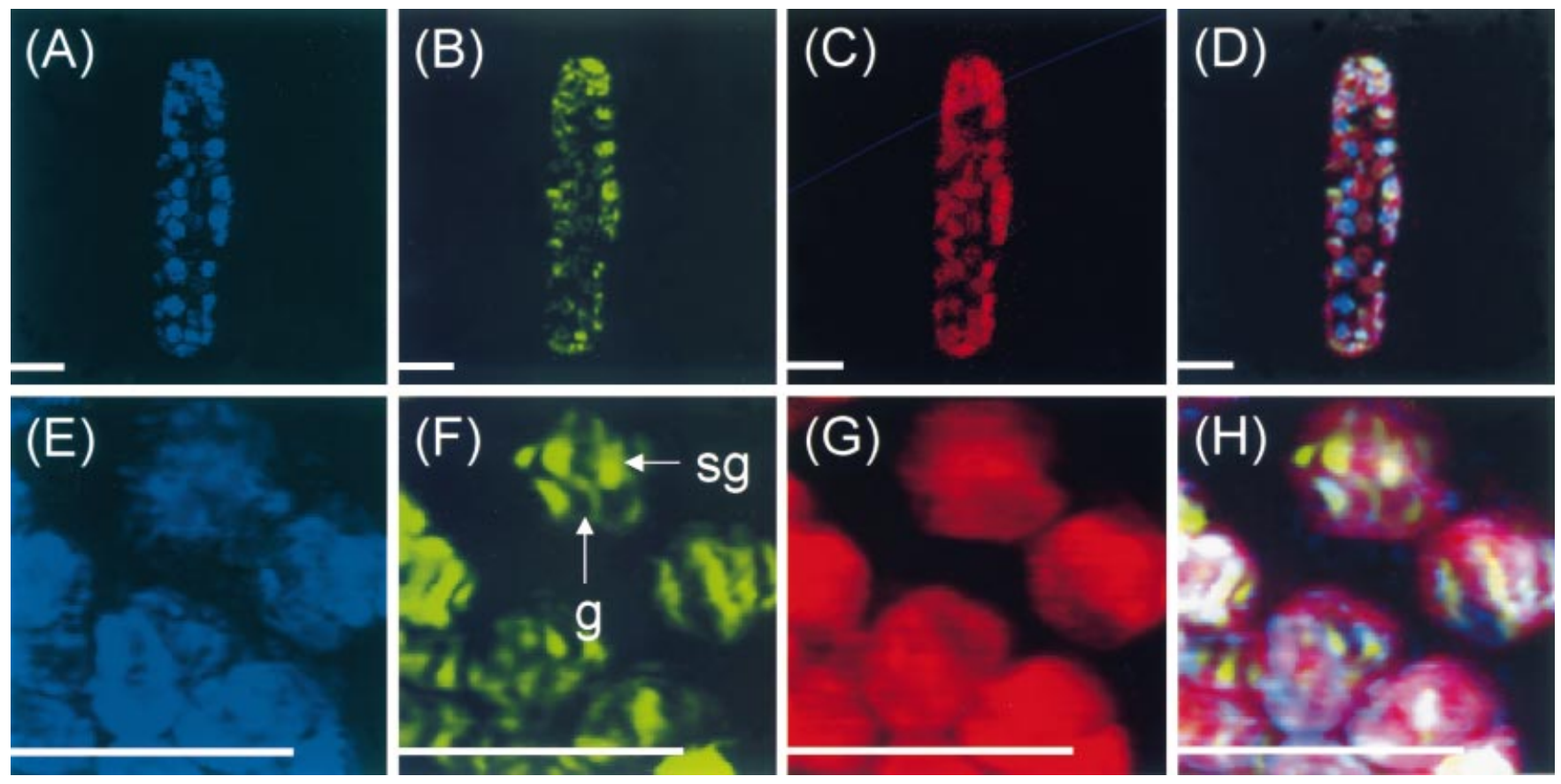

Fig. 10. (A)-(D) Scanning $x-y$ - $\lambda$ images in a live mesophyll cell from Commelina communis L. revealing the distribution of chloroplasts. (E)-(H) Enlarged scanning images showing the individual chloroplasts. By comparing the SHG image with TEM images of similar samples, the crescent-shaped objects in SHG images are identified to be grana and the oval-shaped structures are starch granules. Scale bar: $15 \mu \mathrm{m}$. sg: starch granule. g: grana.

\section{Acknowledgement}

This work was supported by grants from National Science Council (NSC90-2215-E-002-039 and NSC91-2215-E-002021 to C.K.S., NSC89-2311-B-001-134 and NSC89-2311-B001-137 to B.L.L., NSC89-2811-E-002-0058 to P.C.C.) and Academia Sinica (to B.L.L.).

\section{References}

Anderson, R.R. \& Parrish, J.A. (1981) The optics of human skin. J. Invest. Dermatol. 77, 13-19.

Barad, Y., Eisenberg, E., Horowitz, M. \& Silberberg, Y. (1997) Nonlinear scanning laser microscopy by third harmonic generation. Appl. Phys. Lett. 70, 922-924.

Berger, V. (1998) Nonlinear photonic crystals. Phys. Rev. Lett. 81, 4136-4139.

Bouma, B.E., Tearney, G.J., Bilinsky, I.P., Golubovic, B. \& Fujimoto, J.G. (1996) Self-phase-modulated Kerr-lens mode-locked Cr: forsterite laser source for optical coherence tomography. Opt. Lett. 21, 18391841.

Broderick, N.G.R., Ross, G.W., Offerhaus, H.L., Richardson, D.J. \& Hanna, D.C. (2000) Hexagonally Poled lithim niobate: a two-dimensional nonlinear photonic crystal. Phys. Rev. Lett. 84, 4345-4348.

Campagnola, P.J., Wei, M.-D., Lewis, A. \& Loew, L.M. (1999) High resolution nonlinear optical imaging of live cells by second harmonic generation. Biophys. J. 77, 3341-3349.

Chen, I.-H., Chu, S.-W., Sun, C.-K., Lin, B.-L. \& Cheng, P.C. (in press) Wavelength dependent damage in biological multi-photon confocal microscopy: a micro-spectroscopic comparison between femtosecond Ti: sapphire and Cr: forsterite laser sources. Optical Quantum Elect. in press.

Cheng, P.C., Pan, S.J., Shih, A., Kim, K.S., Liou, W.S. \& Park, M.S. (1998) Highly efficient upconverters for multiphoton fluorescence microscopy. J. Microsc. 189, 199-212.

Cheng, P.C., Sun, C.-K., Kao, F.-J., Lin, B.-L. \& Chu, S.-W. (2001) Nonlinear multi-modality spectro-microscopy: multiphoton fluorescence, SHG and THG of biological specimen. SPIE Proc. 4262, 98-103.

Chu, S.-W., Chen, I.-H., Liu, T.-M., Cheng, P.-C., Sun, C.-K. \& Lin, B.-L. (2001) Multi-modal nonlinear spectral microscopy based on a femtosecond Cr: forsterite laser. Opt. Lett. 26, 1909-1911.

Clays, K., Elshocht, S.V., Chi, M., Lepoudre, E. \& Persoons, A. (2001) Bacteriorhodopsin: a natural, efficient (nonlinear) photonic crystal. J. Opt. Soc. Am. B, 18, 1474-1482.

Denk, W., Strickler, J.H. \& Webb, W.W. (1990) Two-photon laser scanning fluorescence microscopy. Science, 248, 73-76.

Dumeige, Y., Vidakovic, P., Sauvage, S., Levenson, J.A., Sibilla, C., Centini, M., D'Aguanno, G. \& Scalora, M. (2001) Enhancement of second-harmonic generation in a one-dimensional semiconductor photonic band gap. Appl. Phys. Lett. 78, 3021-3023.

Freund, I., Deutsch, M. \& Sprecher, A. (1986) Connective tissue polarity: optical second-harmonic microscopy, crossed-beam summation, and small-angle scattering in rat-tail tendon. Biophys. J. 50, 693-712.

Gallant, D.J., Bouchet, B. \& Baldwin, P.M. (1997) Microscopy of starch: evidence of a new level of granule organization. Carbodydrate Polymers, 32, 177-191.

Gannaway, J.N. \& Sheppard, C.J.R. (1978) Second-harmonic imaging in the scanning optical microscope. Opt. Quantum Electron. 10, 435439. 
Gunning, B. \& Steer, M. (1996) Plant Cell Biology: Structure and Function, Jones and Bartlett Publishers, Sudbury, MA, USA, p. 21.

Guo, Y., Ho, P.P., Tirksliunas, A., Liu, F. \& Alfano, R.R. (1997) Optical harmonic generation from animal tissues by the use of picosecond and femtosecond laser pulses. Opt. Lett. 22, 1323-1325.

Hodson, M.J. \& Sangster, A.G. (1989) Silica deposition in the inflorescence bracts of wheat (Triticum aestivum). II, X-ray microanalysis and backscattered electron imaging. Can. J. Bot. 67, 281-287.

Lin, B.-L., Cheng, P.C. \& Sun, C.-K. (2001) Optical density of leaf. Maize Genet. Cooperation News Letters, 75, 61-62.

Liu, T.M., Chu, S.W., Sun, C.K., Lin, B.L., Cheng, P.C. \& Johnson, I. (2001) Multiphoton confocal microscopy using a femtosecond $\mathrm{Cr}$ : forsterite laser. Scanning, 23, 249-254.

Mizutani, G., Sonoda, Y., Sano, H., Sakamoto, M., Takahashi, T. \& Ushioda, S. (2000) Detection of starch granules in a living plant by optical second harmonic microscopy. J. Lumin. 87-89, 824826.

Moreaux, L., Sandre, O., Blanchard-Desce, M. \& Mertz, J. (2000) Membrane imaging by simultaneous second-harmonic generation and two-photon microscopy. Opt. Lett. 25, 320-322.

Müller, M., Squier, J., Wilson, K.R. \& Brakenhoff, G.J. (1998) 3D microscopy of transparent objects using third-harmonic generation. J. Microsc. 191, 266-274.

Peleg, G., Lewis, A., Linial, M. \& Loew, L.M. (1999) Nonlinear optical measurement of membrane potential around single molecules at selected cellular sites. Proc. Natl. Acad. Sci. 96, 6700-6704.

Peter, H.R., Ray, F.E. \& Susan, E.E. (1992) Biology of Plants. Worth Publishers, New York, p. 36.

Shen, Y.R. (1989) Surface properties probed by 2nd harmonic and sum frequency generation. Nature, 337, 519-525.

Sheppard, C.J.R. \& Shotton, D.M. (1997) Confocal Laser Scanning Microscopy. BIOS Scientific Publisher, Oxford, UK.

Sun, C.-K., Chu, S.W., Tai, S.P., Keller, S., Abare. A., Mishira U.K. \& DenBaars, S.P. (2001) Mapping piezoelectric-field distribution in gallium nitride with scanning second-harmonic generation microscopy. Scanning, 23, 182-192.

Sun, C.-K., Chu, S.-W., Tai, S.-P., Keller, S., Mishra U.K. \& DenBaars, S.P. (2000) Scanning second-harmonic-generation and third-harmonicgeneration microscopy of GaN. Appl. Phys. Lett. 77, 2331-2333.

Zhao, T., Chen, Z.-H., Chen, F., Shi, W.-S., Lu, H.-B. \& Yang, G.-Z. (1999) Enhancement of second-harmonic generation in $\mathrm{BaTiO}_{3} / \mathrm{SrTiO}_{3}$ superlattices. Phys. Rev. B, 60, 1697-1700. 